\title{
Time Based Global Track Reconstruction in CBM
}

\author{
Timur Ablyazimov ${ }^{1,2, \star}$, Volker Friese ${ }^{1, \star \star}$, and Victor Ivanov ${ }^{2,3, \star \star \star}$ \\ ${ }^{1}$ GSI Helmholtzzentrum für Schwerionenforschung GmbH, Darmstadt, Germany \\ ${ }^{2}$ Laboratory of Information Technologies, Joint Institute for Nuclear Researches, Dubna, Russia \\ ${ }^{3}$ National Research Nuclear University "MEPhl”, Moscow, Russia
}

\begin{abstract}
The paper describes the approaches, implemented by the authors, for the track reconstruction in data, subdivided to time slices, in the CBM experiment.
\end{abstract}

\section{Introduction}

CBM ("Compressed Baryonic Matter") [1] is an experiment being prepared to operate at the future Facility for Anti-Proton and Ion Research (FAIR) in Darmstadt, Germany. The CBM experiment will explore the high-density region of the QCD phase diagram by investigating nuclear collisions from 2 to $45 \mathrm{GeV}$ beam energy per nucleon. Its main focus is the measurement of very rare probes (e.g. charmed hadrons), which requires interaction rates of up to $10 \mathrm{MHz}$. A specific feature of the experiment will be the free-running data acquisition fashion, without a hardware trigger. It differs from the traditional event based approach, and demands taking into account the time-coordinate measurements in track reconstruction, and using smart approaches to cope with an enormous combinatorics. For the sake of brevity, and also because the time measurements are taken into account in the described reconstruction algorithms, this approach is further referred as "time based". Physical studies in the CBM experiment are planned to be based on the the so called "global" track reconstruction - which means the reconstructed tracks comprise data, obtained from different detectors. The CBM software is developed under the CBMROOT framework, which in turn extensively uses the CERN ROOT infrastructure. Before the authors started their work, the only time based track reconstruction component in the CBMROOT software repository was the track finder for the Silicon Tracking System (STS) detector, called L1. It was also available a facility for the global tracking, called Littrack, in the CBMROOT software package, but it was not optimized for work with timely sliced data - it treated input data from time slices the same way as for events, which, presumably, gave it only limited reconstructing capabilities. The local reconstruction - clustering - was also implemented without taking into account time for the most of the CBM detecting systems. The main goal of authors' efforts was to link measurements from the Time of Flight (ToF) detector to the reconstruction chain. There were two possible directions of development: adapting the existing global tracking software (Littrack) to the time slice based reconstruction and creating a dedicated ToF hits linking software from scratch. When the work started, there was no working measurements clustering software for ToF, optimized to work with time

\footnotetext{
$\star$ e-mail: t.ablyazimov@gsi.de

$\star \star$ e-mail: v.friese@gsi.de

$\star \star \star$ e-mail: ivanov@jinr.ru
} 
slices. So the first step was to implement a clustering algorithm for ToF, taking time into account. As a final result both global track reconstruction approaches have also been implemented.

\section{Measurements clustering in ToF}

Before global (track) reconstruction can be done, the local entities, hits, have to be reconstructed in all the involved detectors. There were already time based cluster finders for the STS and the Muon Chamber $(\mathrm{MuCh})$ detectors, but was no such a finder for ToF. So it was implemented by the authors. ToF detector is a "wall", consisting of detecting "bricks" - Multi-gap Resistive Plate Chambers (MRPCs) - of several different types, having nevertheless common conceptual structure, which is depicted on the figure 1.

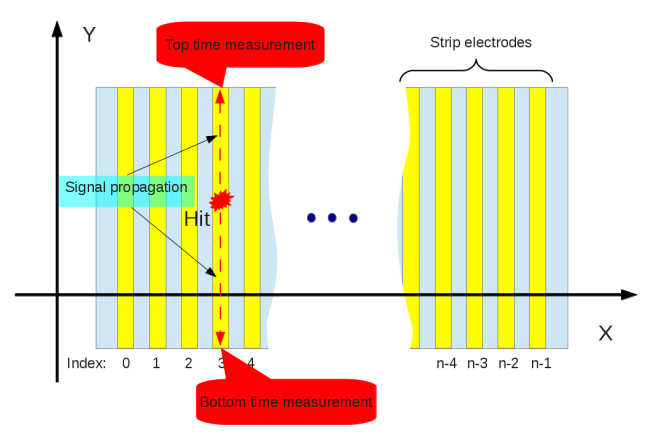

Figure 1. ToF measurements are obtained on the top and the bottom ends of the ToF MRPC conducting strips, and provide time information with high precision

ToF hits are represented as points in a 4-dimensional space: 3 spatial coordinates, and one time coordinate. The later coordinate is obtained in the most straightforward way - as an average of the corresponding top and bottom time measurements. One spatial coordinate ("horizontal" - X) is calculated in the MRPC local frame of reference from the measurement strip number and the known MRPC pitch. Another ("vertical" - Y) is calculated by the difference between measurements on the top and the bottom of an MRPC channel. The last space coordinate $(\mathrm{Z})$ is determined by the position of an MRPC self, and X and Y coordinates of a point inside the MRPC. Measurements from different neighbouring MRPC channels are clustered together, because a single particle can fire multiple channels at once.

\section{Dedicated ToF tracker}

This tracker uses tracks, reconstructed in STS, extrapolating them into the area of the ToF wall. Then it tries to link them with the ToF hits, located in the vicinity. The time coordinate is taken into account in the vicinity definition. The track extrapolation uses the routines, implemented in the Littrack library. In the initial version of the dedicated tracker the time was handled separately, but, after upgrading Littrack (as described in the next section), the time was added as another component of the state vector, describing the dynamical model of tracking. To keep combinatorics on moderate level in situations, when time slices contain much of data, the ToF measurements data are subdivided to addressable 
space-time regions of moderate size - cuboids. The ToF station self is represented as a bigger cuboid in the 4-dimensional space-time. The time span is equal to the time slice duration. The spans in space corresponds to the dimensions of the 3-dimensional cuboid, circumscribing the ToF detector. The detecting station cuboid is subdivided to the smaller cuboids, used then for the acceleration of hit searches, as shown of the figure 2. The smaller cuboids, used for hit searches, are later referred as "bins".

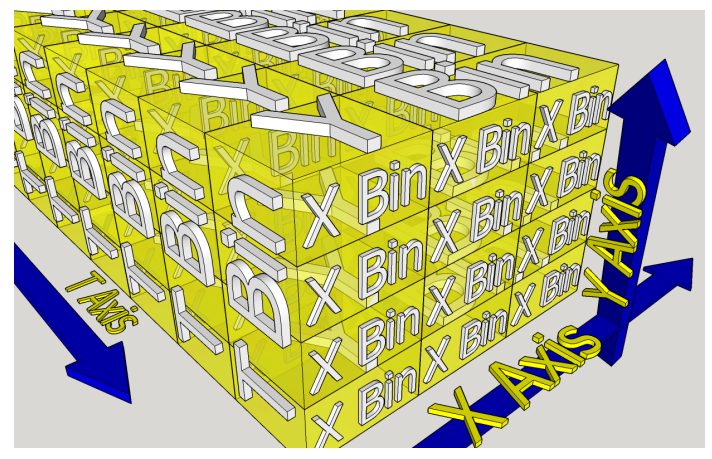

Figure 2. Hierarchical binning in 3D space

As the influence of the magnetic field is negligible in the ToF wall area, the tracks are modeled as a straight lines. It makes the process of locating bins appropriate to a particular track easy: these bins are bins, which are intersected by the track, as shown on the left half of the figure 3. All the intersected bins are inspected for hits, and the closest with respect to all space and time coordinates hit is assigned to the track (if its distance from the track, measured in the units of errors for space and time coordinate, is not too big) - this is depicted on the right side of the same figure 3 .
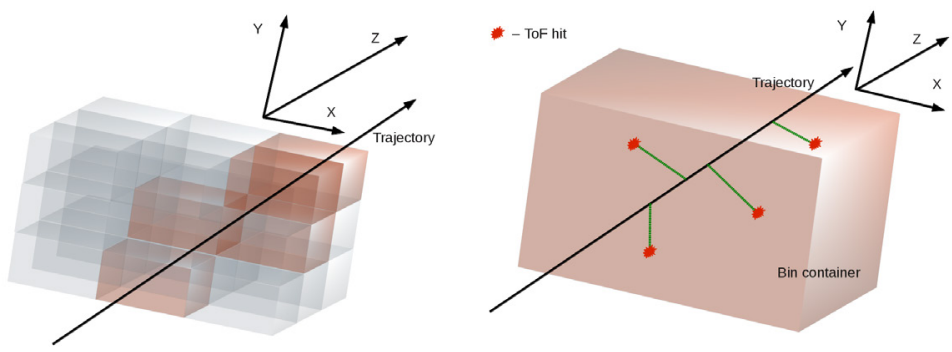

Figure 3. Selecting a subset of bins for a track and selecting the closest hit in this subset

\section{Littrack upgrade}

The Littrack library is available in the CBMROOT framework for many years and intended for reconstructing tracks from measurements, obtained concurrently from several different detecting systems (global tracking) [2]. Track reconstruction in the Littrack library is based on the track following approach and uses Kalman filter formalism for calculation of particles physical properties. It uses track parameters from the L1 track finder as seeds. One of the detectors, which data the Littrack library can use in its reconstruction chain, is ToF. Because of its important role, it was decided to try to upgrade 
the Littrack library for the time based reconstruction as well. It was done, by carefully upgrading the underlying Kalman's formalism: time coordinate was added to the state vector, the appropriate members were added to the covariance matrix, and the Runge-Kutta method implementation was upgraded with calculation for the time related terms. One of the other detectors, supported by the Littrack library, is MuCh. The currently existing clustering algorithm in $\mathrm{MuCh}$ is also able to work with time based data. So Littrack has been tested for the ability to use MuCh and ToF hits in the time based reconstruction.

\section{Results}

All the three described here software components were tested with respect to their efficiency. The first two of them were also tested for their performance. The testing was conducted on an Intel(R) Xeon(R) CPU E5-1607 v3 @ 3.10GHz machine. For the tests, where only ToF hits were linking to reconstructed L1 tracks, input data, produced with URQMD generator, simulating minimum bias $\mathrm{Au}+\mathrm{Au}$ collisions at $10 \mathrm{AGeV}$, were used. The Littrack efficiencies were tested with input data, produced by the Box generator, simulating a pure stream of muons (20 muons per event), having energies in the range 4-10 GeV. Monte Carlo tracks were considered reconstructible according the criterion of having at minimum the following number of points in detecting systems: 4 in STS, 4 in $\mathrm{MuCh}$ and 1 in Tof - the efficiencies were calculated for this kind of tracks. The matching criterion fulfilled, a reconstructed track had at least $70 \%$ common points with a Monte Carlo. The results are presented in the table 1, where the figures for reconstruction in time slices are compared with the efficiencies of the appropriate tools in the event by event mode.

Table 1. Algorithms testing results

\begin{tabular}{lll}
\hline Algorithm & Efficiency (\%) & Runtime (per event) \\
\hline ToF clustering (time slices) & 97 & $4 \mathrm{~ms}$ \\
Dedicated ToF tracker (time slices) & 93 & $14 \mathrm{~ms}$ \\
Littrack (ToF) (time slices) & 93 & N/A \\
Littrack (MuCh) (time slices) & 98 & N/A \\
Littrack (MuCh+ToF) (time slices) & 89 & N/A \\
Littrack (ToF) (event by event) & 93 & N/A \\
Littrack (MuCh) (event by event) & 100 & N/A \\
Littrack (MuCh+ToF) (event by event) & 86 & N/A
\end{tabular}

It can be seen that the Littrack reconstruction efficiency was a little bit worse for MuCh $(98 \%$ vs nearly $100 \%$ ). Probably the main reason for this was the inconsistency between the MuCh detector simulation, where the electronics delay was taken into account, and the MuCh hit reconstruction, where this delay was neglected. The mentioned delay was about $17 \mathrm{~ns}$ in average. It was partially compensated by shifting the time measurement values during loading the MuCh input date into Littrack by $17 \mathrm{~ns}$. But the correct solution must be later done in the MuCh hit finder.

\section{References}

[1] B. Friman, P. Senger et al., The CBM Physics Book (Lecture Notes in Physics, Vol. 814, 1st Edition, 2011) $960 \mathrm{pp}$.

[2] A. Lebedev, The Software and Algorithms for the Track Reconstruction in the Transition Radiation and the Muon Chamber Detectors in the CBM Experiment (Ph.D. thesis, Dubna, 2011) $97 \mathrm{pp}$. 